\title{
CORPUS ÉLECTRONIQUES DE L'ANCIEN ÉGYPTIEN: TRAITEMENT XML DES TEXTES DES PROCESSIONS DE SOUBASSEMENT DES TEMPLES TARDIFS
}

\author{
Vincent Razanajao
}

\begin{abstract}
An important element of the grammar of the temple, the processions of Nile Gods inscribed on the lower parts of walls in temples of Ptolemaic and Roman date form an interesting corpus for the study of toponyms or for the history of religions. When the author came to consider the form the publication of this corpus should take, the idea of an electronic publication and treatment by XML seemed very promising.

The aim of this paper is not to elaborate the Document Type Definition (DTD) dedicated to Egyptian texts but to put forward the author's comments subsequent to having used the corpus. The subject of the project being textual corpora, the approach will follow that of the Text Encoding Initiative (TEI). After a brief bistory of processions and an analysis of their structure, a series of adjustments of the TEI tags will be presented with an explanation of the bias chosen. The outline of an XML file is appended at the end of the paper.
\end{abstract}

Les réflexions d'ordre informatique dont je souhaite faire part dans les quelques pages qui vont suivre sont nées des questionnements apparus lors de l'établissement d'un projet d'édition des textes des processions ornant les soubassements des temples tardifs, principalement d'époques grecque et romaine. Lorsqu'il s'est agi de réfléchir à la forme que devait prendre ce corpus, l'idée d'une publication électronique a tout de suite paru opportune et, 
plus précisément, un traitement par XML de ces textes a semblé tout à fait intéressant.

L'aspect électronique répond à la volonté d'aller au-delà de la publication des textes pour elle-même, l'objectif étant de développer un outil permettant une interrogation multiple et illimitée de la matière contenu dans ces textes. La possibilité serait ainsi offerte à tout chercheur d'obtenir aisément, par exemple, l'ensemble des textes relatifs à la province d'Égypte sur laquelle il travaille.

\section{LE XML, SES PRINCIPES ET SES APPLICATIONS DANS LES ETUDES DE CORPUS TEXTUELS}

\section{Principes généraux du XML}

Le XML, ou «eXtensible Markup Language», a pour principe d'augmenter toute chaîne de caractères - et donc tout texte - de «balises» invisibles permettant d'ajouter des informations sur les mots qui la composent. Par exemple, le balisage XML de la notice «égyptologie» du Petit Robert consisterait à indiquer que:

-le premier mot, «égyptologie», est l'entrée de la notice;

$-«$ mil. XIX ${ }^{\mathrm{e} »}$ est la date de la première occurrence;

-«de Égypte et -logie» constitue l'étymologie;

-«étude scientifique de l'Égypte ancienne»en donne le sens.

Les balises XML étant de petites séquences entre signes $<$ et $>$, une traduction en XML de cet exemple donnerait:

$<$ mot $><$ entrée $>$ Égyptologie $<$ /entrée $>$, $<$ date $>$ mil. XIX ${ }^{\mathrm{e}}</$ date $>$, $<$ étymologie $>$ de Égypte et -logie $</$ étymologie $>$. $<$ définition $>$ Étude scientifique de l'Égypte ancienne $</$ définition $>$. $</$ mot $>$

\section{Les règles de saisie $\mathrm{XML}$}

Le traitement d'un texte selon les normes XML passe par la définition préalable du canevas et des règles qui seront utilisés pour la saisie. L'ensemble de ces règles est répertorié dans ce qui s'appelle une DTD, ou «Document Type Definition», une sorte de document modèle qui énonce les règles propres à la mise en XML des textes du corpus correspondant. 


\section{XML et corpus textuels: les préceptes de la TEI}

Traiter de manière informatique le corpus qui nous intéresse nécessite de s'inscrire dans les problématiques développées par la TEI, ou Text Encoding Initiative (http:/ /www.tei-c.org/), «que l'on pourrait traduire, ainsi que cela a été très justement écrit ${ }^{1}$, par groupe d'initiative pour le balisage normalisé des textes» et qui constitue «une norme de balisage, de notation et d'échange de corpus des documents électroniques» ${ }^{2}$. La TEI, par la publication régulière de recommandations ${ }^{3}$, propose un large panel de balises XML qu'il s'agit d'adapter aux besoins de son propre objet d'étude.

Loin d'être contraignante, cette nécessité de prendre comme assise les jeux de balises et autres composants XML définis dans la TEI permet de rendre intelligibles les corpus entre chercheurs. De surcroît, si le but premier de la TEI est de permettre et de faciliter l'échange des corpus eux-mêmes, un autre atout de suivre la TEI est que cela offre une possibilité d'analyser et de confronter les approches en elles-mêmes. S'inscrire dans la logique TEI permet ainsi de s'appuyer sur l'expérience acquise dans l'élaboration des corpus de textes électroniques. Pour notre propos, notre regard doit également se porter sur les groupes de travail qui ont plus particulièrement pour objet la publication en ligne des textes épigraphiques ou en langues anciennes. Au premier rang de ces groupes de travail, il faut citer EpiDoc, ou Epigraphic Documents in TEI XML (http://epidoc.sourceforge.net/), et le Perseus Project (http://www.perseus.tufts.edu/), deux émanations de la TEI particulièrement intéressantes pour notre réflexion.

L'objectif du présent article étant moins de définir la DTD propre aux textes égyptiens que de soumettre les quelques conclusions qu'il m'a paru possible de tirer de mes interrogations sur le corpus des textes des processions des temples tardifs, il s'agit désormais de se pencher plus avant sur ces textes qui s'organisent selon une structure qui sied particulièrement à un traitement XML.

1 L. Romary, H. Hudrisier, «TEI - Text Text Encoding Initiative», article en ligne sur le site Internet du RIFAL, Réseau international francophone d'aménagement linguistique, http://www.culture.gouv.fr/culture/dglf/rifal/tei.htm (dernière consultation: octobre 2008).

2 Ibid.

3 Nous en sommes actuellement à la cinquième édition: http://www.teic.org/Guidelines/P5/ (dernière consultation: octobre 2008). 


\section{LES PROCESSIONS DE SOUBASSEMENT DES TEMPLES TARDIFS: HISTOIRE ET TYPOLOGIE}

Élément important de la grammaire du temple, les processions de figures ornant les soubassements des temples d'époques ptolémaïque et romaine constituent un corpus dont la richesse s'avère particulièrement intéressante en ce qui concerne non seulement les études toponymiques mais également l'histoire des religions ou celle, plus générale, des mentalités.

\section{Les processions dans l'histoire}

Dès le début de la $\mathrm{IV}^{\mathrm{e}}$ dynastie et le règne de Snéfrou ${ }^{4}$, des personnages incarnant les provinces $(s p 3 \cdot t)$ d'Égypte apparaissent dans l'ornementation des parois des temples funéraires royaux aux côtés des figurations des domaines du défunt venus lui apporter les richesses de ses terres. Les processions de personnages androgynes ou féminins seront dès lors le support privilégié pour représenter le territoire et ses richesses. Glissant du monde des défunts à celui des dieux, les défilés viendront enrichir progressivement l'iconographie des temples. Cette évolution est plus particulièrement intéressante à suivre en ce qui concerne les processions d'ordre géographique.

Ce n'est cependant pas sous l'aspect d'une procession qu'apparaît la première énumération géographique connue produite dans le domaine religieux non-funéraire. C'est en effet sous la forme d'une liste disposée en tableau qu'apparaît le dénombrement de sépats que le roi Sésostris $\mathrm{I}^{\text {er }}$ a fait figurer sur le soubassement de sa Chapelle blanche à Karnak ${ }^{5}$. Plus tard, sur la Chapelle rouge érigée à Karnak par Hatchepsout - monument dont la fonction est identique à celle de la Chapelle blanche -, le tableau des sépats ornant le soubassement est remplacé par une procession de figures androgynes et féminines ${ }^{6}$. Si l'information fournie par ce collège de personnages est bien moins importante puisqu'elle ne se résume guère qu'au seul nom de la province, la charge symbolique peut être considérée comme bien plus importante puisque ce retour à la procession proprement figurée renoue

4 A. FAKHry, The Monuments of Snefru at Dashur. II. The Valley Temple. Part I. The Temple Reliefs, Le Caire, 1961, p. 17-58.

5 P. Lacau, H. Chevrier, Une chapelle blanche de Sésostris $I^{e r}$, Le Caire, 1956, p. 207-251.

6 Id., Une chapelle d'Hatchepsout à Karnak, [Le Caire], 1977, p. 69-92. 
avec une forme plastique finalement beaucoup plus expressive que le tableau.

Comme souvent dans l'iconographie égyptienne, le texte vient soutenir l'image, mais quoique connaissant huit variantes ${ }^{7}$, les formules accompagnant les porteurs d'offrandes de la Chapelle rouge d'Hatchepsout restent très stéréotypées et ne présentent que de manière très générique les biens présentés: «Je t’apporte toutes les choses bonnes et douces issues de la Terre du Nord», «toutes les offrandes fraîches et pures», «toutes les provisions», etc. Ce n'est qu'à partir de l'époque ramesside que la nature de ces formules s'étoffe et que celles-ci abandonnent leur aspect stéréotypé, allant parfois même jusqu'à évoquer des spécificités régionales propres ${ }^{8}$. À cette même époque, les processions présentant des figures incarnant les produits et les richesses du pays connaissent également un regain d'intérêt?

Connues par des modèles remontant à l'époque Sheshonquide ${ }^{10}$, les processions de «Nils» gravées sur les soubassements des temples d'époque ptolémaïque puis romaine vont acquérir une réelle épaisseur quant au contenu de leurs textes. Les exemples les mieux conservés sont assurément ceux des temples d'Horus à Edfou et d'Hathor à Dendara, mais rares sont les temples qui ne comportent pas de ces défilés dans leur programme décoratif (Figure 1).

\section{Formes et types des processions en ouvre sur les parois des temples gréco-romains}

Dans cette époque mémorielle où les hiérogrammates puisent dans leurs archives pour compiler le compendium sacré que l'on peut lire aujourd'hui, entre autres, sur les parois des derniers temples, les motivations profondes dans le choix des toponymes et des entités représentées sont complexes ${ }^{11}$. Le sujet de ces processions est tant à la fois géographique - dans le cadre d'une une géographie sacrée-, économique ou mythologique, ces trois domaines se mêlant de manière étroite.

7 P. Lacau, H. CheVrier, Une chapelle d'Hatchepsout à Karnak, [Le Caire], 1977, p. 71.

8 J. YoYOTTE, AEPHE $V^{\mathscr{C}}$ section 98, 1989-1990, p. 179.

9 Voir par exemple la procession du temple de Séthy I ${ }^{\text {er }}$ à Gurna (H. BRUGSCH, Die Geographie des alten Ägytens, Geographische Inschriften altägyptischer Denkmäler 1, Leipzig, 1857, pl. XII).

10 Id., «Note sur le bloc de Sheshonq I découvert par la mission archéologique à Saqqara de l'Université de Pisa», Egitto e Vicino Oriente 12, 1989, p. 33-35. 
En ce qui concerne les processions ornant les soubassements des temples gréco-romains, quatre grandes catégories ont été mises en évidence ${ }^{12}$ :

1) les processions géographiques, lesquelles se décomposent en:

a) Les défilés des seules quarante-deux figures représentant chacune une sépat d'Égypte;

b) Les processions plus développées dites quadripartites, défilés ainsi dénommés parce que chaque province est représentée par quatre personnages, incarnations des quatre composantes d'une province: la sp3.t elle-même, le canal- $m r$ et les territoires $w$ et $p h b$ qui lui sont propres.

2) les processions hydrologiques;

3) une variante de ces dernières où sont mis en scène des couples inondation/campagne;

4) les processions économiques.

\section{Structure générale des textes des processions de soubassement}

Quoique des variations en nuancent l'apparente unicité, ainsi que le montre la typologie rappelée ci-dessus, les textes des processions forment un ensemble cohérent tant du point de vue de la forme que du contenu, ce qui rend particulièrement intéressante leur mise en corpus. Ainsi, en prenant en considération les seules processions géographiques par exemple, un rapide examen permet de voir que les textes de chaque province s'articulent autour de deux syntagmes ${ }^{13}$ : un premier présentant l'entité de la procession et les produits qu'elle apporte avec elle; un second définissant un certain nombre d'assimilations divines destinées à caractériser la divinité récipiendaire en fonction de l'apport (la tradition égyptologique désigne cette seconde partie sous le nom de "glose d'assimilation»). À l'intérieur de ces deux syntagmes, plusieurs composantes grammaticales et sémantiques donnent corps à ce système. Si l'on prend par exemple le texte de la procession concernant la $\mathrm{XIX}^{\mathrm{e}}$ sépat de Basse-Égypte, il est possible de mettre en exergue différents éléments tel que cela est illustré dans la Figure 2.

11 Voir J. YOYOTTE, Orientalia 35, 1966, p. 46; id., AnnEPHE $V^{\mathscr{e}}$ section 75, $1967-$ 1968, p. 106; 91, 1982-1983, p. 217-221; Chr. ZIVIE-COCHE, Tanis. Statues et autobiographies de dignitaires. Tanis à l'époque ptolémaïque, TTR 3, Paris, 2004, p. 294; V. RAZANAjaO, «Le Delta à Basse époque: géographies d'un territoire», Égypte. Afrique et Orient 42, juin 2006, p. 3-9.

12 J. YоуотTE, Annuaire du Collège de France 94, 1993-1994, p. 685-686.

13 Dans le sens large donné à ce mot par F. de Saussure, Cours de linguistique générale, Lausanne, Paris, 1916, p. 172. 


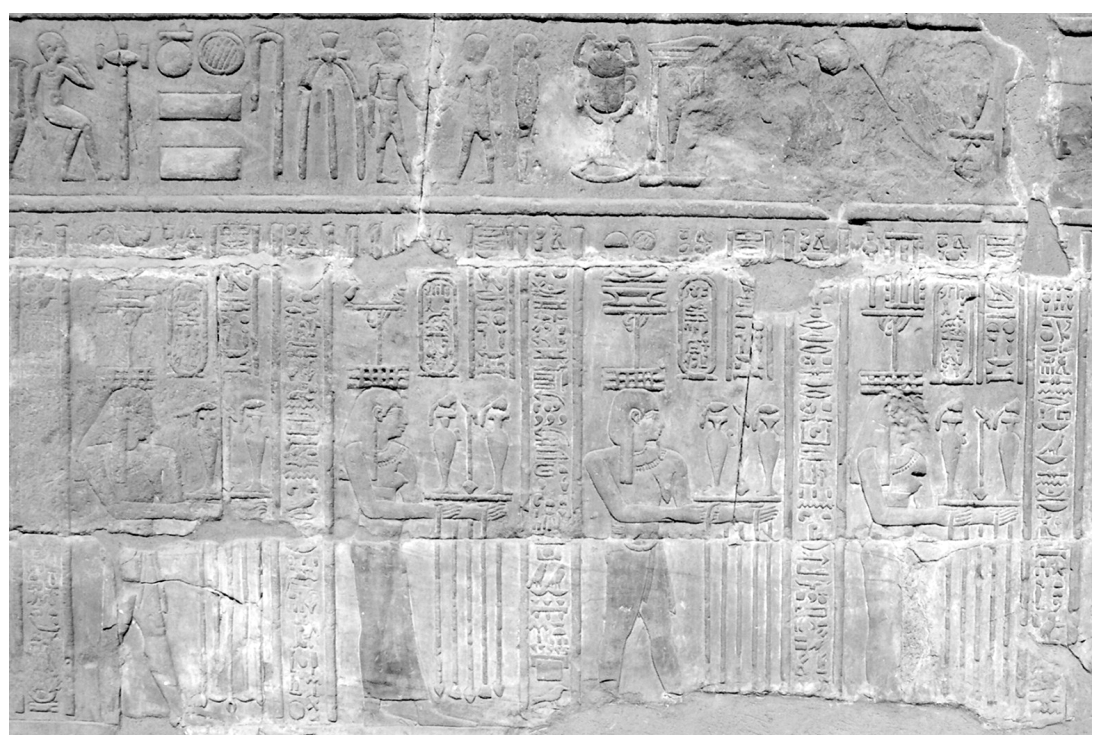

Figure 1: Edfou, soubassement du mur ouest de la cour du temple d'Horus. Début de la procession quadripartite des sépats de Basse-Égypte (C) Photo de l'auteur.

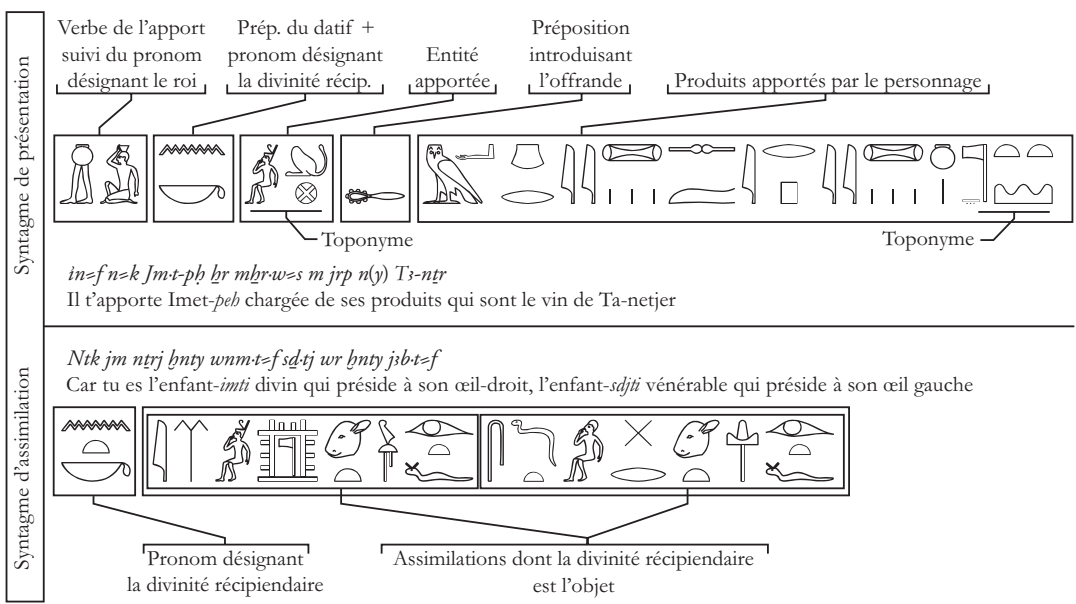

Figure 2: Mise en exergue des éléments composant les textes accompagnant une figure de procession, en l'occurrence la sépat de la XIX ${ }^{\mathrm{e}}$ province de Basse-Égypte (Edfou IV, 37, sqq.) 
Un traitement selon les normes XML du texte de la Figure 2 consisterait à indiquer en premier lieu qu'il est question de la procession ornant le soubassement du pronaos du temple d'Edfou (= Edfou IV, 21-39 pour la Basse-Égypte; IV, 172-193 pour la Haute-Égypte). À un niveau inférieur, il faudrait renseigner le fait qu'il s'agit du texte de la XIX sépat (Edfou IV, 37, $3-38,1)$ puis que de «in=f» à «T3-ntrr», nous avons affaire au syntagme de présentation, et de «ntk» à «j3b.t=f», au syntagme d'assimilation. Par la suite, il est possible de marquer les différents éléments constitutifs du texte, qu'il s'agisse d'objets grammaticaux (préposition introduisant l'apport, pronom introduisant la divinité récipiendaire) ou sémantique (assimilations divines dont la divinité récipiendaire est l'objet). Un balisage XML permettrait également de renseigner le type de personnage qui incarne l'entité apportée, celui de sa coiffe, sa position dans la procession. D'un caractère plus «flottant», un autre balisage aura son importance: celui des toponymes. Pouvant apparaître dans les deux syntagmes, les noms de lieux pourront ainsi être marqués, quelle que soit leur position dans le texte. La Figure 3 donne l'ébauche de la structure générique des processions qu'il parait possible de mettre en avant.

Il reste à s'interroger sur la manière que l'on pourrait user pour traduire en XML ce schéma générique, et quels éléments et balises de la TEI il s'agit de prendre en considération.

\section{POUR UNE DFINITION D'UNE DTD-TEI PROPRE AUX TEXTES ÉGYPTIENS}

Un premier jet de DTD liée aux textes hiéroglyphiques a vu le jour en $2000^{14}$. Comme son auteur S. Rosmorduc l'explique en introduction, cette DTD n'avait pas pour vocation de baliser les textes hiéroglyphiques d'un point de vue sémantique mais bien du seul point de vue formel. La question était centrée sur les problèmes que pose la saisie même des textes hiéroglyphiques et la manière de les encoder, indépendamment presque du XML, suivant les préceptes du Manuel de codage. Mais, et particulièrement dans le cadre qui nous occupe ici-celui des textes des processions de soubassement-, il s'avère que l'intérêt du XML et de l'application des

14 Consultable sur les pages Internet de S. Rosmorduc à l'adresse http://webperso.iut.univ-paris8.fr/ rosmord/HieroEncoding/DTD/ (dernière consultation: octobre 2008). 


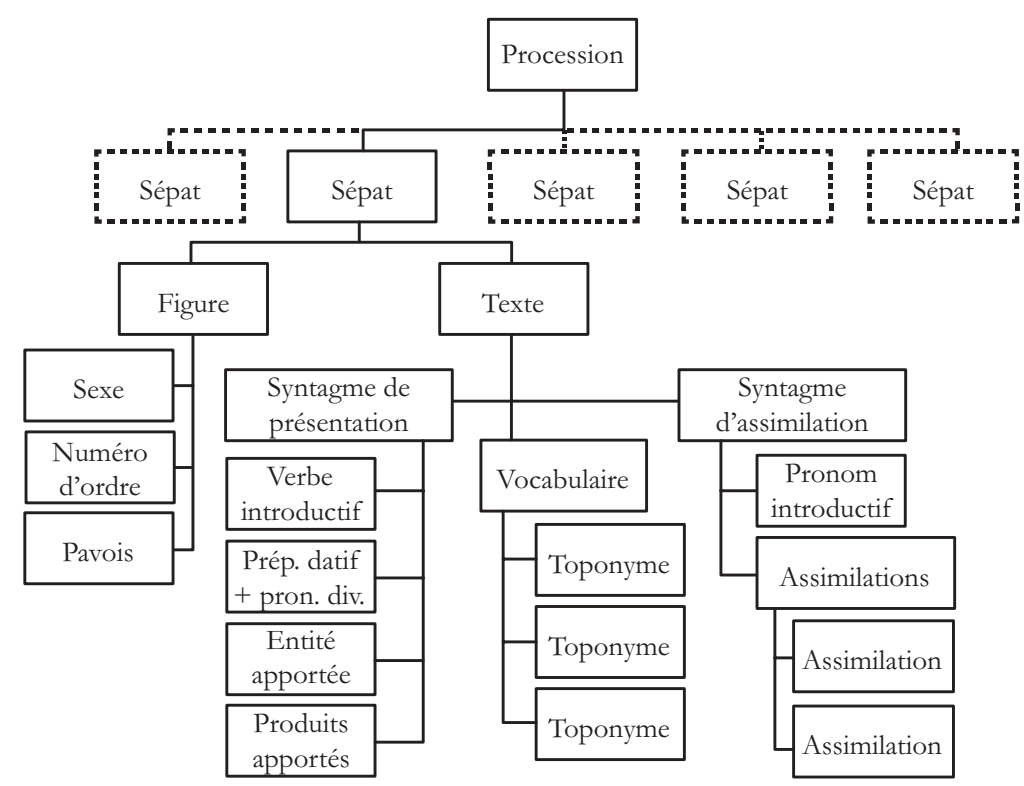

Figure 3: Arborescence de la structure générique applicable aux processions de soubassement

recommandations TEI réside dans les réponses que ceux-ci apportent à la façon dont on peut appréhender un corpus textuel sur le plan sémantique.

Malgré les apparentes dissemblances qui séparent les textes égyptiens des manuscrits médiévaux ou modernes - principal sujet d'études des corpus TEI -, il semble possible d'affirmer que l'ensemble des entités et balises contenues dans la TEI répondent aux besoins, à l'instar de ce qui a été fait dans le cadre du projet EPIDOC. Aucune création de balise n'est nécessaire car l'adaptation de celles existantes par le biais des définitions d'attributs est très souple et extensible.

Dès l'abord, il apparaît que les entités définies par la TEI afin de renseigner les informations générales sur un manuscrit et destinées à figurer dans l'en-tête fonctionnent parfaitement. Le «header» du fichier XML sur les processions pourra donc reprendre les éléments de la TEI suivant: $<$ filedesc> (file description) contient la description du fichier électronique d'un point de vue bibliographique. 
$<$ profilDesc> (text-profile description) contient la description du texte contenu dans le fichier électronique du point de vue de son élaboration, de son contexte de production, de sa langue (balises <settingDesc>, <creation>, etc.).

$<$ ClassDecl> (classification declarations) répertorie les références qui seront utilisées pour des renvois externes au document. Par exemple, il est possible de définir les abréviations bibliographiques qui seront utilisées tout au long du corpus (Dendara, Edfou, $W b$, PM, etc.).

Afin de baliser la structure générale de chaque texte relatif à une figure, des $\langle$ div $>$ avec trois «types» différents pourraient être utilisées:

$<$ div type="presentation">, division concernant les informations sur la figure elle-même (genre, numéro d'ordre, pavois);

$<$ div type="presentation" $>$, division concernant les textes du syntagme de présentation;

$<$ div type="presentation">, division concernant les textes du syntagme d'assimilation;

À l'intérieur de chacune de ces divisions, il s'agit de pouvoir saisir des hiéroglyphes, de la translittération et de la traduction. Les $<$ div $>$ suivantes pourraient être utilisées:

<div type="texthi"> pour les hiéroglyphes;

$<$ div type="textal"> pour la translittération $\left(« a l »=\left\langle\right.\right.$ alphabetic $\left.{ }^{15}\right)$;

$<$ div type="texttr"> pour la traduction.

Ces dernières $\langle$ div $>$ étant d'un niveau inférieur à celui des $<$ div type="presentation" $>$, etc., l'utilisation de «div» hiérarchisées sera un avantage. Ainsi, <div1> pour les grandes parties de texte, <div2> pour les séquences hiéroglyphiques, de translittération et de traduction.

L'adaptation de l'existant permet également de ne pas avoir à créer de balises particulières pour l'encodage des signes hiéroglyphiques. Il nous parait ainsi envisageable d'utiliser les balises suivantes:

$<g>\quad$ (character or glyph) pour le balisage d'un signe hiéroglyphique unique.

15 Les suffixes «hi», «al» et «tr» sont repris de M. J. NEDERHOF, «Alignment of Resources of Ancient Egyptian Texts Based on XML», dans Proceedings of the 14th Table Ronde Informatique et égyptologie, Pise, 2002, ressource en ligne: http://www.cs.st-andrews.ac.uk/ mjn/egyptian/align/index.html, p. 2 (dernière consultation: octobre 2008). 
<seg> (segment) permettrait de procéder aux regroupements de signes. Une définition adéquate de l'attribut type permettrait d'indiquer le type de regroupement: cadrat ou, à l'intérieur d'un cadrat, groupe horizontal, vertical, ou encore ligature.

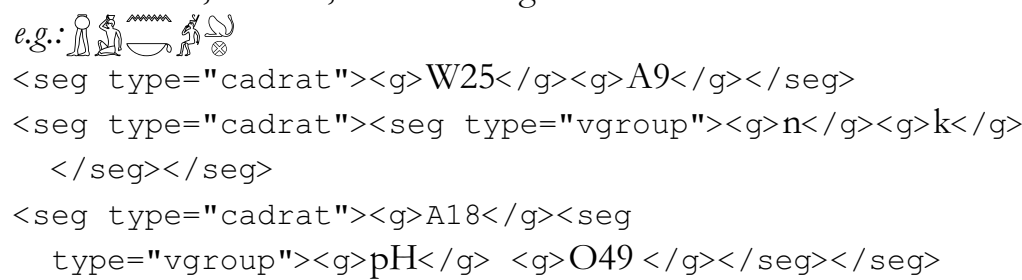

L'indication de hachure pourrait être portée sur un texte par l'utilisation de la balise <unclear> dont le type serait défini comme «hatch». Les balises TEI <sic $>$, <corr $>$, <add $>$, <del>, le couple <lacunastart $>/<$ lacunaEnd $>$ (ou la balise $<$ gap $>$ ?) et $<$ supplied $>$ permettront de porter toutes les indications utiles à l'édition du texte.

En ce qui concerne la translittération et la traduction, diverses balises permettront d'affiner l'encodage sémantique des mots et séquences qui composent le texte:

$<w>\quad$ (word) ainsi que la définit la TEI, «represents a grammatical (not necessarily orthographic) word». Cette balise permettra par exemple de marquer un toponyme. L'attribut «lemma» sera utile pour préciser la forme exacte du mot.

e.g.: le balisage de la translittération de pourrait être rendu par:

$<$ w type="toponym" lemma="Jm.t-ph" $>J m \cdot k$ unclear $>-p h$

$</$ unclear $\rangle\langle$ w $\rangle$

$<r s>$ (referencing string), permettrait de marquer, par exemple, que la

partie du texte ainsi balisée concerne les produits apportés.

e.g.: cf. texte du syntagme d'assimilation de la Figure 2.

$<$ w type="pronoun_assim" $>n t k</$ w $>$

$\langle$ rs type="assimilation" $>j m$ ntrj bnty $w n m \cdot t=f\langle$ rs $\rangle$

$<r s$ type="assimilation" $>$ s $\underline{d} \cdot t j$ wr bnty $j 3 b \cdot t=f<|r s\rangle$

Comme on peut le voir, les préceptes établis par la TEI répondent aux besoins de l'égyptologue en matière de traitement d'un corpus textuel. Je ne saurais prétendre, par cet article, établir une DTD-TEI propre aux textes égyptiens, ceci nécessitant un évident travail en commun. Aussi, je n'approfondirai pas plus la description des adaptations qu'il m'a paru possible de mener sur les balises XML-TEI. J'ai toutefois annexé en fin d'article une ébauche de fichier XML qui permet, je l'espère, d'illustrer la manière dont il parait possible d'aborder les textes égyptiens. 
Les réflexions dont je vous ai fait part ont été induites par la nature même du corpus que je souhaitais traiter, celui des textes des processions de soubassement des temples tardifs. Cet article se veut avant tout l'annonce de ce projet et une pierre dans l'établissement d'une DTD pour les textes en ancien égyptien. Il apparaît nécessaire, pour l'avenir, d'établir un groupe de travail à l'image d'EPIDOC, groupe de travail égyptologique qui aurait sa place toute trouvée dans le cadre des tables rondes Égyptologie \& informatique.

\section{ANNEXE. ÉBAUCHE DE FICHIER XML-TEI POUR L'ENCODAGE DES TEXTES DES PROCESSIONS DE SOUBASSEMENT DES TEMPLES TARDIFS}

\begin{tabular}{|c|c|}
\hline 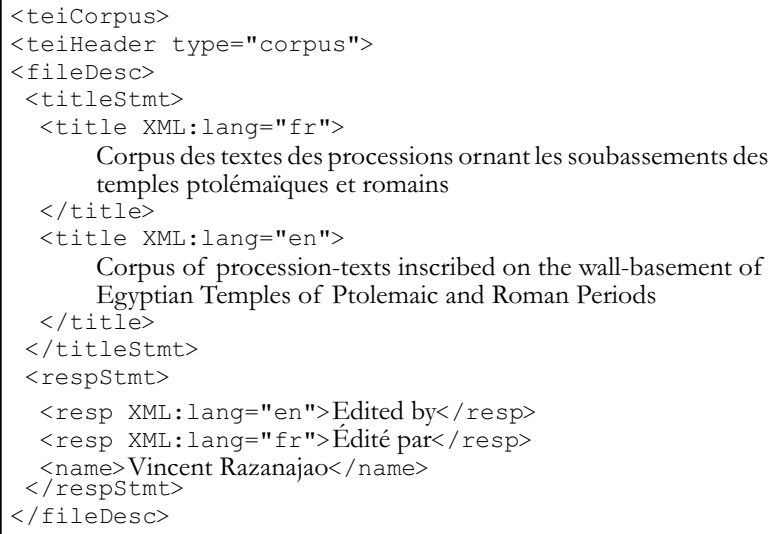 & $\begin{array}{l}\text { En-tête de fichier avec le } \\
\text { titre du corpus dans son } \\
\text { entier; } \\
\text { Filedesc: description } \\
\text { générale du fichier XML, } \\
\text { avec indication de l'éditeur } \\
\text { (respStmt); }\end{array}$ \\
\hline 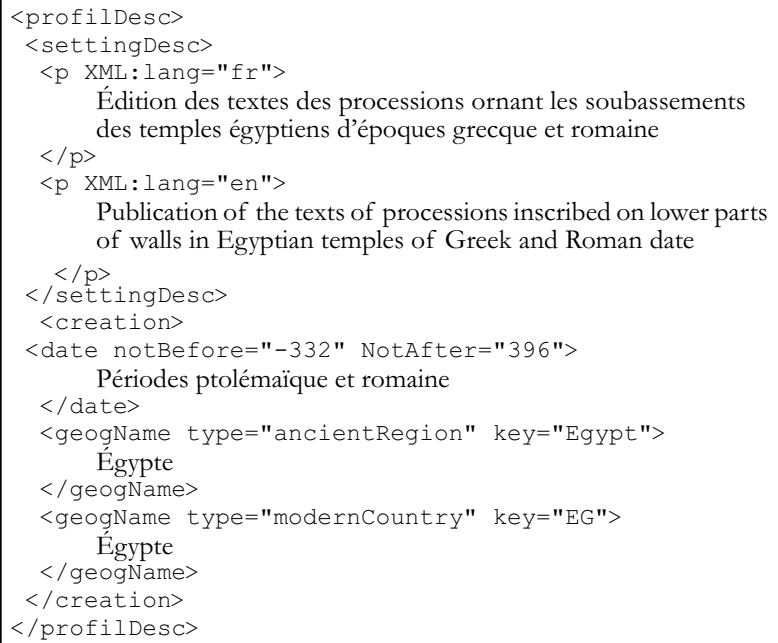 & $\begin{array}{l}\text { Description générale du } \\
\text { corpus, avec indications de } \\
\text { dates et de localisation } \\
\text { (creation); }\end{array}$ \\
\hline
\end{tabular}




\begin{tabular}{|c|c|}
\hline 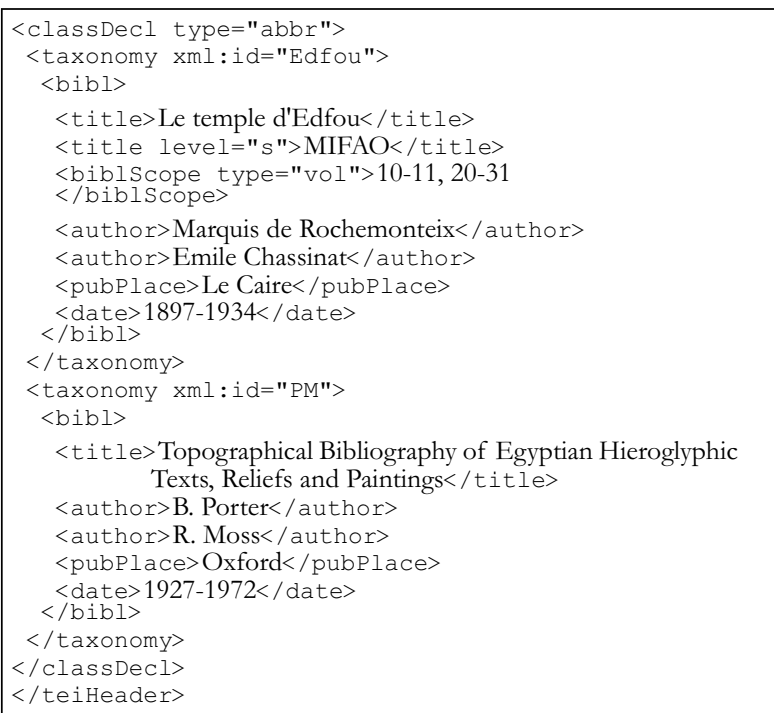 & $\begin{array}{l}\text { Définition des abréviations } \\
\text { (taxonomy) } \\
\text { bibliographiques qui seront } \\
\text { utilisées dans le corpus (ici, } \\
\text { définition de "Edfou" et } \\
\text { "PM"). } \\
\text { - title level="s": titre de } \\
\text { collection }\end{array}$ \\
\hline 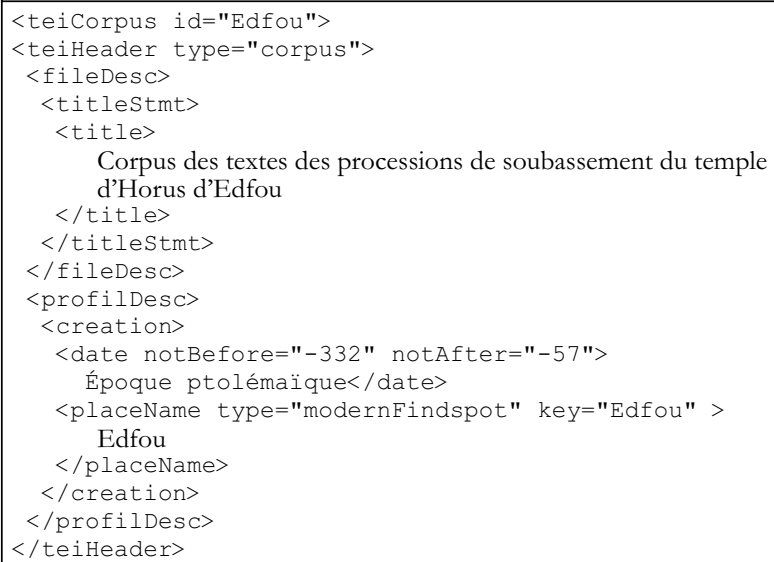 & $\begin{array}{l}\text { Début du sous-corpus } \\
\text { rassemblant les textes du } \\
\text { temple d'Horus d'Edfou, } \\
\text { avec les informations } \\
\text { relatives aux dates et } \\
\text { localisation (profilDesc). }\end{array}$ \\
\hline $\begin{array}{l}\text { <teiCorpus id="Edfou_corridor_ext"> } \\
\text { <teiHeader type="corpus"> } \\
\text { <fileDesc> } \\
\text { <titlestmt> } \\
\text { <title> } \\
\quad \text { Procession du corridor extérieur } \\
\text { </title> } \\
\text { </titleStmt }> \\
\text { <sourceDesc }> \\
\text { <ref target="\#Edfou"> } \\
\text { Edfou IV, 21-42,170-194 } \\
\text { </ref }> \\
\text { </sourceDesc }> \\
\text { </fileDesc }>\end{array}$ & $\begin{array}{l}\text { Début du sous-corpus des } \\
\text { textes ornant le corridor } \\
\text { extérieur du temple } \\
\text { d'Edfou. } \\
\text { - ref target permet } \\
\text { d'utiliser l'abréviation } \\
\text { définie plus haut; }\end{array}$ \\
\hline
\end{tabular}




\begin{tabular}{|c|c|}
\hline $\begin{array}{l}\text { <profilDesc> } \\
\text { <creation> } \\
\text { <date notBefore="-204" notAfter="-181"> } \\
\text { Ptolemy V Épiphane et Cléopâtre III } \\
\text { </date> } \\
\text { <placeName type="temple" key="Horus_Edfou"> } \\
\text { Temple d'Horus à Edfou } \\
\text { </placeName> } \\
\text { <placeName type="room" key="corridor_ext"> } \\
\text { Corridor extérieur } \\
\text { </placeName> } \\
\text { </creation> } \\
\text { </profilDesc } \\
</ \text { teiHeader }>\end{array}$ & \\
\hline $\begin{array}{l}<\text { <ei.2 n="BE01"> } \\
<\overline{\text { tei.2> }}\end{array}$ & $\begin{array}{l}\text { Textes relatifs à la première } \\
\text { sépat de Basse-Égypte. }\end{array}$ \\
\hline & $\begin{array}{l}\text { Textes concernant les } \\
\text { sépats } 2 \text { à } 18\end{array}$ \\
\hline 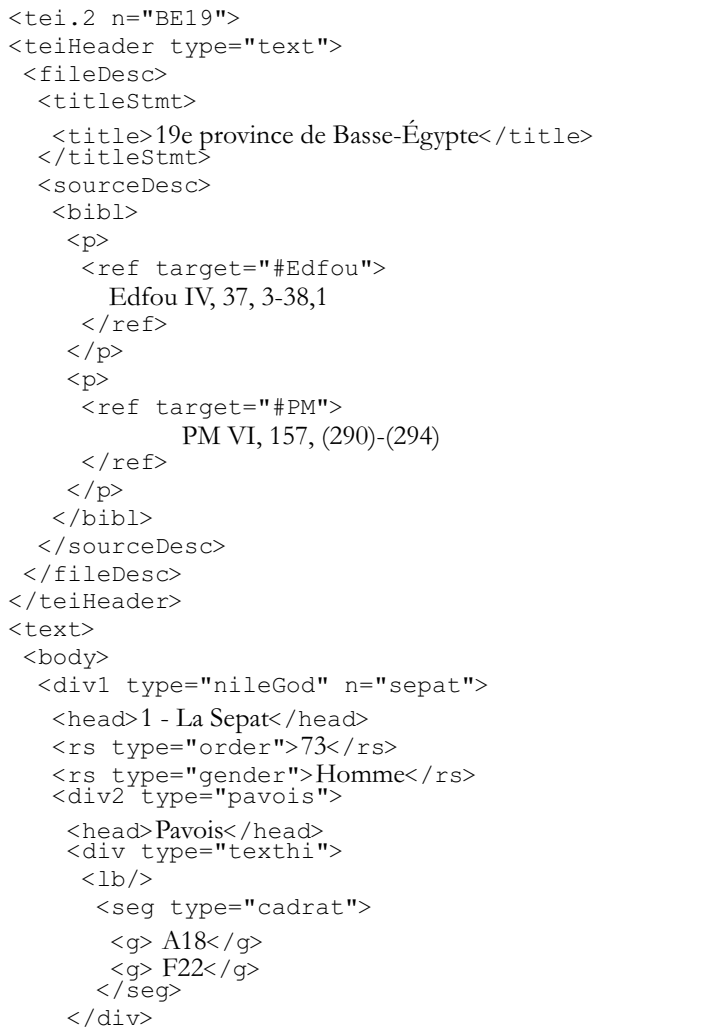 & $\begin{array}{l}\text { Textes de la sépat elle-même; } \\
\text { A. Section relative au } \\
\text { personnage lui-même, } \\
\text { indications (rs) sur: } \\
\text { - le sexe; } \\
\text { - numéro d'ordre dans la } \\
\text { procession; } \\
\text { et indication sur ce que } \\
\text { porte le pavois (div } \\
\text { type="pavois"); }\end{array}$ \\
\hline
\end{tabular}




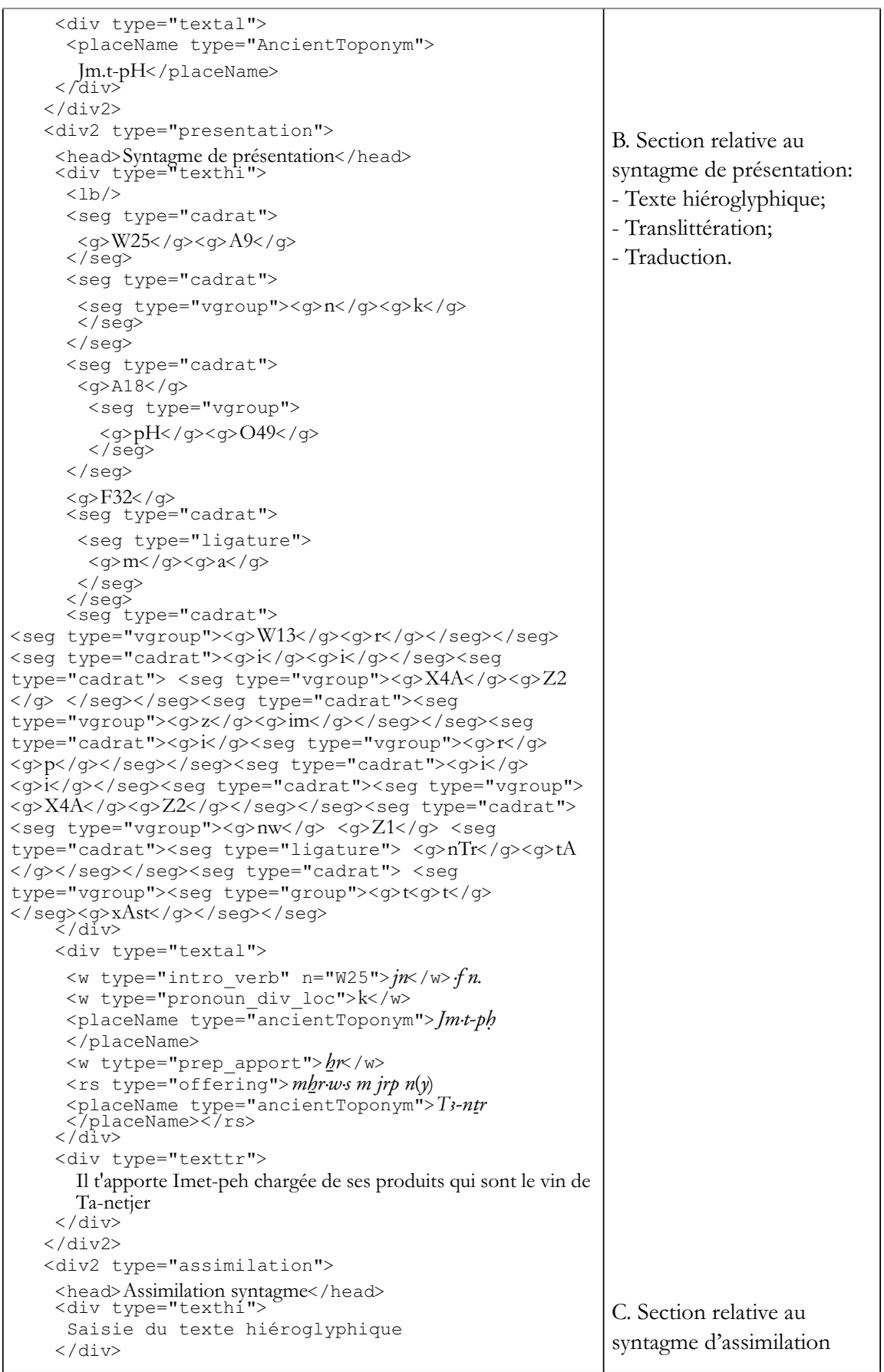




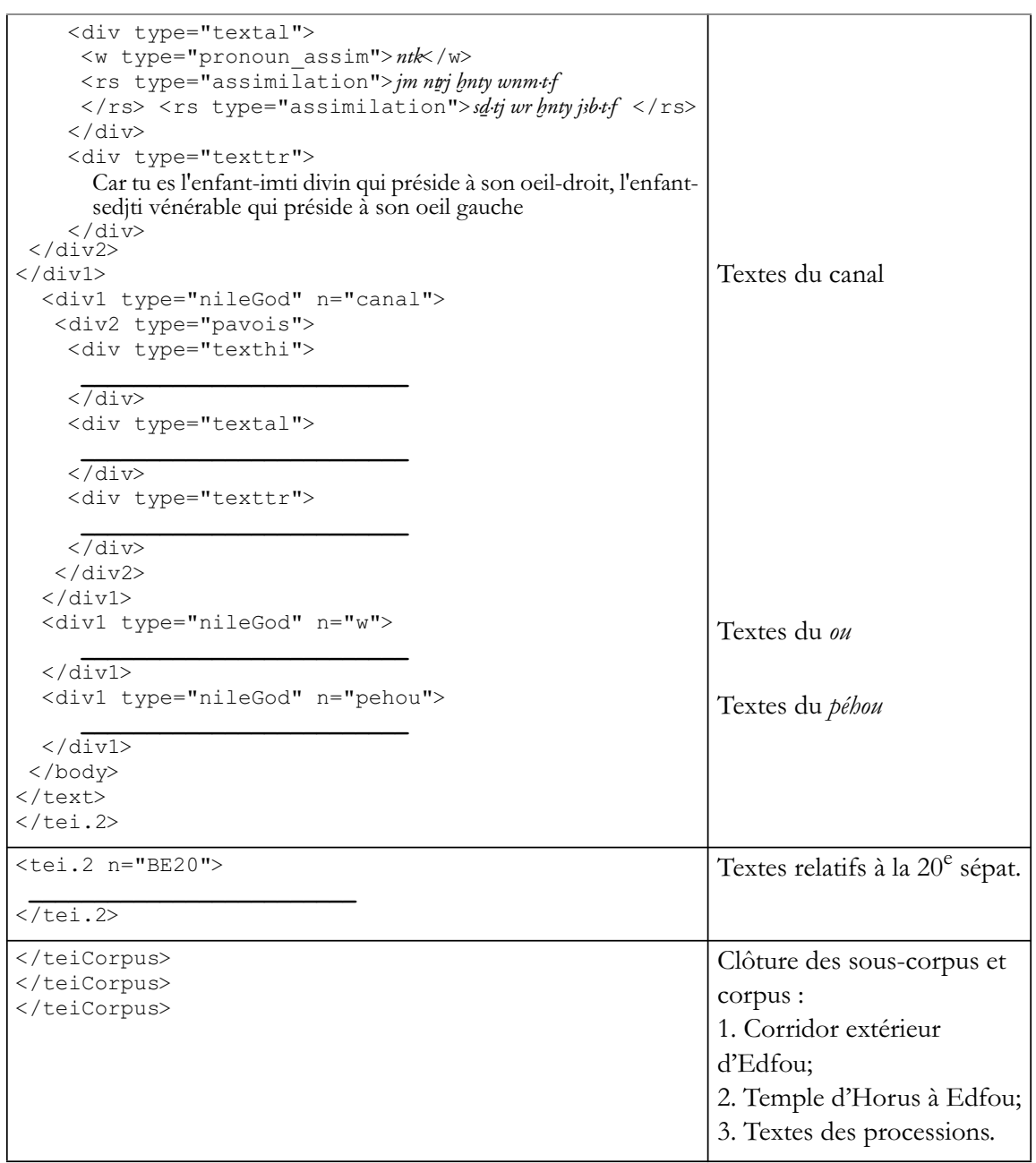

